\title{
Sleep and pain: a circadian multi-challenge rather than a simple bidirectional pathway
}

\author{
Sono e dor: um multi-desafio circadiano, mais do que uma simples via bidirecional
}

Dear Editor,

The relationship between sleep and pain was recently revisited by Whibley et al. ${ }^{1}$ who aimed to provide additional evidence on the mutual interaction between those functional states of life. But something is missing, so we think.

Sleep and pain lay down in common structural and functional matrixes. However, neither anatomical pathways or neurochemical substrates may entirely explain the complex interaction which subsides the physiopathology and clinical features of comorbid sleep impairment with acute or chronic pain. Interestingly, while acute pain experience may develop a state of increased arousal from which an individual becomes ready to run away from the real or potential physical injury, chronic pain often involves a state of rest in which the individual tries to become protected from the harmful stimulus. In both cases, the behavior seems to reflect a protective instinct which had probably contributed to make sleep/wake cycle and pain a succeeded evolutionary interactive trait. In the clinical scope, this relationship is highly important as it mutually affects sleep and pain-related disturbances and impacts the prognosis of many disorders co-occurring with any of those conditions.

Both sleep and pain are functions of a well-orchestrated multi-oscillatory mechanism characterizing the Circadian Timing System (CTS), with a central clock and several peripheral oscillators collectively known as peripheral clocks. These complex and self-maintained machinery dictates an internal time for virtually all our physiology ${ }^{2}$. Therefore, it is reasonable to accept an interdependent process between those vital functions, which in turn mutually feed in a circadian fashion favoring a multidimensional clinical outcome.

Although sleep-related circadian regulation is perfectly established, there is a lack of good quality studies on circadian mediation of either pain and sleep-pain interaction, thus leading to a misinterpretation of the true directional dynamics. Insufficient or inadequate sleep is known to contribute to increased pain intensity and a reduction of pain tolerance. CTS plays a fundamental role in sleep propensity, which was early integrated and reappraised in an explanatory model for sleep regulation ${ }^{3}$. On the other hand, pain either in idiopathic form or associated with a primary condition may negatively impact sleep in all its domains depending on the circadian moment ${ }^{4}$. Some important features of pain are indeed clock-dependent even if is not defined whether those rhythms are derived from daily oscillations within the underlying causes driving the pain or from the rhythmic oscillatory component of the neural processing of pain. Finally, chronotype is also a signature on pain-related oscillatory mechanisms regardless of the social time 5 . Understanding the basics of such temporally guided processes may provide not only a deeper knowledge of the interactive physiological mechanisms between sleep and pain but also an important background to clinical decisions. Furthermore, the multidimensional features of circadian modulatory systems within the distinct mechanisms of pain makes their interconnection a must in any kind of analytic approach of sleep-pain interaction turning the CTS a factor to include in a future review model based on mediation analysis.

Keywords: Circadian rhythm, Circadian rhythm sleep disorders, Pain, Sleep disorders.

Conflict of interests: none - Sponsoring sources: none

Miguel Meira e Cruz https://orcid.org/0000-0001-6076-0878 Izabella Paola Manetta

(D) https://orcid.org/0000-0001-6641-0713

Universidade de Lisboa, Faculdade de Medicina, Centro Cardiovascular, Unidade do Sono,Lisboa, Portugal. E-mail:mcruz@medicina.ulisboa.pt

(c) Sociedade Brasileira para o Estudo da Dor 


\section{REFERENCES}

1. Whibley D, AlKandari N, Kristensen K, Barnish M, Rzewuska M, Druce KL, et al. Sleep and pain: a systematic review of studies of mediation. Clin J Pain. 2019;35(6):544-58.

2. Meira E Cruz M, Miyazawa M, Manfredini R, Cardinali D, Madrid JA, Reiter R, et al. Impact of daylight-saving time on circadian timing system: an expert statement.
Eur J Intern Med. 2019;60:1-3.

3. Borbély AA, Daan S, Wirz-Justice A, Deboer T. The two-process model of sleep regulation: a reappraisal. J Sleep Res. 2016;25(2):131-43.

4. Hagenauer MH, Crodelle JA, Piltz SH, Toporikova N, Ferguson P, Booth V. The modulation of pain by circadian and sleep-dependent processes: a review of the experimental evidence. 2017. doi: http://dx.doi.org/10.1101/098269.

5. Jankowski KS. Morning types are less sensitive to pain than evening types all day long. Eur J Pain. 2013;17(7):1068-73. 